\title{
Universiteit
}

Leiden

The Netherlands

\section{Determinant quantum Monte Carlo study of the screening of the one- body potential near a metal-insulator transition}

Chakraborty, P.B.; Denteneer, P.J.H.; Scalettar, R.T.

\section{Citation}

Chakraborty, P. B., Denteneer, P. J. H., \& Scalettar, R. T. (2007). Determinant quantum Monte Carlo study of the screening of the one-body potential near a metal-insulator transition.

Physical Review B, 75(12), 125117. doi:10.1103/PhysRevB.75.125117

Version: $\quad$ Not Applicable (or Unknown)

License: $\quad$ Leiden University Non-exclusive license

Downloaded from: https://hdl.handle.net/1887/77045

Note: To cite this publication please use the final published version (if applicable). 


\title{
Determinant quantum Monte Carlo study of the screening of the one-body potential near a metal-insulator transition
}

\author{
P. B. Chakraborty, ${ }^{1}$ P. J. H. Denteneer, ${ }^{2}$ and R. T. Scalettar ${ }^{1}$ \\ ${ }^{1}$ Physics Department, University of California, Davis, California 95616, USA \\ ${ }^{2}$ Lorentz Institute, LION, Leiden University, P.O. Box 9506, 2300 RA Leiden, The Netherlands \\ (Received 20 October 2006; revised manuscript received 23 January 2007; published 26 March 2007)
}

\begin{abstract}
In this paper, we present a determinant quantum Monte Carlo study of the two-dimensional Hubbard model with random site disorder. We show that, as in the case of bond disorder, the system undergoes a transition from an Anderson insulating phase to a metallic phase as the on-site repulsion $U$ is increased beyond a critical value $U_{c}$. However, there appears to be no sharp signal of this metal-insulator transition in the screened site energies. We observe that, while the system remains metallic for interaction values up to twice $U_{c}$, the conductivity is maximal in the metallic phase just beyond $U_{c}$ and decreases for larger correlation.
\end{abstract}

DOI: 10.1103/PhysRevB.75.125117

PACS number(s): 71.10.Fd, 71.30.+h, 02.70.Uu

\section{INTRODUCTION}

The metal-insulator transition arising from the competition of randomness and interactions remains an intriguing problem in condensed-matter physics. For example, the question of the existence of a metallic phase in two dimensions, for which an experimental consensus had emerged in the $1980 \mathrm{~s},{ }^{1}$ has been revisited with new samples over the last decade, with developments which have driven a considerable amount of new theoretical work. ${ }^{2,3}$

Several interesting lines of study have emerged, which explore the interplay of one-body potentials and two-body interactions in more general contexts. The superfluidinsulator transition has been studied in disordered, interacting boson systems, where the existence of a thermodynamic order parameter, the superfluid density $\rho_{s}$, as well as the greater ease of numerical simulations, has resulted in many definitive results. ${ }^{4-6}$ The coexistence of a metal and a MottHubbard insulating phase in the disordered half-filled Hubbard model has been explored using both numerical and analytical techniques. ${ }^{7}$ The existence of insulating phases away from commensurate fermion filling has been explored in models with bimodal distributions of on-site chemical potential. ${ }^{8,9}$ Finally, the question of metallic phases arising from the addition of correlations to a band insulator is drawing new attention. ${ }^{10}$

The commonly cited qualitative picture of the appearance of a metallic phase out of a disordered one is that the interactions act to screen the one-body potential. While several quantum Monte Carlo studies of disordered interacting fermions exist, ${ }^{11-13}$ which demonstrate the possibility of a metallic phase, none have looked quantitatively at this screening in the Hubbard Hamiltonian.

In this paper, we will present results for the conductivity and renormalized site energy of the two-dimensional Anderson-Hubbard model,

$$
\begin{aligned}
H= & -t \sum_{\langle j l\rangle, \sigma}\left(c_{j \sigma}^{\dagger} c_{l \sigma}+c_{l \sigma}^{\dagger} c_{j \sigma}\right)+U \sum_{j} n_{j \uparrow} n_{j \downarrow} \\
& +\sum_{j}\left(\epsilon_{j}-\mu\right)\left(n_{j \uparrow}+n_{j \downarrow}\right) .
\end{aligned}
$$

Here, $c_{j \sigma}^{\dagger}\left(c_{j \sigma}\right)$ are fermion creation (destruction) operators on site $j$ for spin $\sigma$ and $n_{j \sigma}=c_{j \sigma}^{\dagger} c_{j \sigma}$ is the number operator. $t$ is the hopping parameter, $U$ the onsite repulsion, and $\mu$ and $\epsilon_{j}$ the global chemical potential and local site energies, respectively. Each $\epsilon_{j}$ is drawn independently from a uniform distribution on $\left(-\frac{1}{2} \Delta,+\frac{1}{2} \Delta\right)$. We choose $t=1$ to set our scale of energy.

Our key conclusion is that while increasing $U$ can drive an Anderson insulating phase metallic, there appears to be no sharp signature of this transition in the variance of the renormalized site energies. This suggests that the metallic phase arises at least partially from an additional mechanism beyond a simple screening of the one-body potential.

\section{NUMERICAL APPROACH}

We employ the determinant quantum Monte Carlo (DQMC) method. ${ }^{14}$ Since many descriptions of the approach exist, we only provide a brief sketch here, focusing on those features most relevant to the present study. DQMC is an exact method to compute the properties of tight-binding Hamiltonians on finite lattices. The inverse temperature $\beta$ in the partition function is discretized, and an auxiliary ("Hubbard-Stratonovich") field is introduced to decouple the interactions. The resulting quadratic form in fermion creation and destruction operators is integrated out analytically, leaving a sum over the Hubbard-Stratonovich variables, which can be performed stochastically. ${ }^{15}$

We have chosen the imaginary-time discretization size small enough such that the systematic "Trotter" errors are comparable to the statistical errors associated with the Monte Carlo sampling and disorder averaging. Of greater concern in these simulations is the finite lattice size and, in particular, the possibility of a "false" signal of metallic behavior, which would occur if the localization length exceeds the lattice size. We have verified that in the phases we identify as metallic, the localization length (computed at $U=0$ ) is less than the lattice size.

To investigate the metal-insulator transition, we look directly at the dc conductivity, which we obtain from the current-current correlation function

$$
j_{x}(\ell, \tau)=e^{H \tau}\left[i t \sum_{\sigma}\left(c_{\ell+\hat{x}, \sigma}^{\dagger} c_{\ell \sigma}-c_{\ell \sigma}^{\dagger} c_{\ell+\hat{x}, \sigma}\right)\right] e^{-H \tau} .
$$

We compute the Fourier transform $j_{x}(\mathbf{q}, \tau)$ of $j_{x}(\ell, \tau)$ and its correlation function $\Lambda_{x x}(\mathbf{q}, \tau)=\left\langle j_{x}(\mathbf{q}, \tau) j_{x}(-\mathbf{q}, 0)\right\rangle$. Using the 


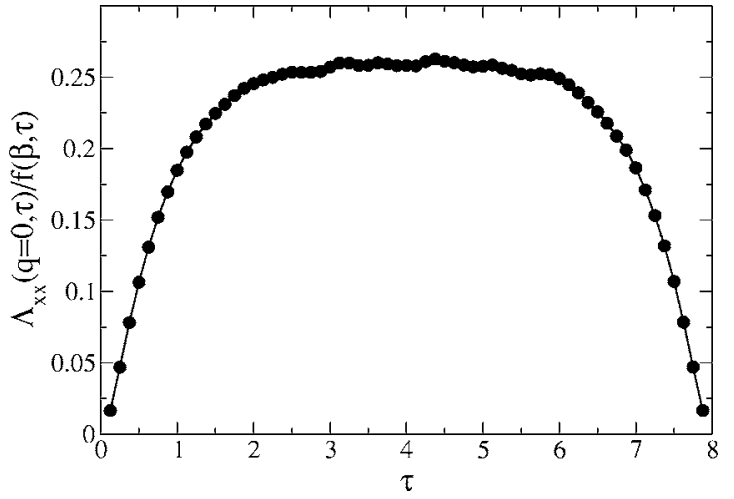

FIG. 1. A plot of the ratio between the imaginary-time currentcurrent correlation function at $\mathbf{q}=0$ and the function $f(\beta, \tau)$ as defined in the text. The parameters are $U=2, \Delta=8$, and $\beta=8$. The result is an average of ten disorder realizations. The ratio $\Lambda_{x x}(\mathbf{q}=0, \tau) / f(\beta, \tau)$ will fluctuate more around $\tau=\beta / 2$ for lower $T$ and larger $U$, but not in such a way to invalidate our approximation for $\sigma_{\mathrm{dc}}$. The linear lattice size is 8 .

general formalism of linear-response theory, the dc conductivity is given by

$$
\sigma_{\mathrm{dc}}=\lim _{\omega \rightarrow 0} \frac{\operatorname{Im} \Lambda_{x x}(\mathbf{q}=0, \omega)}{\omega} .
$$

The frequency-dependent conductivity is given by the integral transform

$$
\Lambda_{x x}(\mathbf{q}, \tau)=\int_{-\infty}^{\infty} \frac{d \omega}{\pi} \frac{e^{-\omega \tau}}{1-e^{-\beta \omega}} \operatorname{Im} \Lambda_{x x}(\mathbf{q}, \omega) .
$$

It is difficult to obtain $\operatorname{Im} \Lambda_{x x}(\mathbf{q}, \omega)$ by inverting this integral equation, because it requires the determination of $\Lambda_{x x}(\mathbf{q}, \tau)$ on a very fine mesh of imaginary times $\tau$ with very high numerical accuracy. However, if we insert $\tau=\beta / 2$ in Eq. (4), the function multiplying $\operatorname{Im} \Lambda_{x x}(\mathbf{q}, \omega)$ for low $T$ effectively restricts the integral to small $\omega$, so that we may approximate $\operatorname{Im} \Lambda_{x x}(\mathbf{q}=0, \omega)$ by $\sigma_{\mathrm{dc}} \omega$. The validity of the above approximation can be checked by inserting Eq. (3) in Eq. (4): if the approximation of linear response is valid, then Eq. (4) becomes

$$
\Lambda_{x x}(\mathbf{q}=0, \tau)=\sigma_{\mathrm{dc}} \int_{-\infty}^{\infty} \frac{d \omega}{\pi} \frac{\omega e^{-\omega \tau}}{1-e^{-\beta \omega}}=\sigma_{\mathrm{dc}} f(\beta, \tau) .
$$

This implies that the ratio $\Lambda_{x x}(\mathbf{q}=0, \tau) / f(\beta, \tau)$ should be independent of $\tau$ near $\tau=\beta / 2$. In Fig. 1, we show this ratio against $\tau$ for a representative set of parameters, averaged over ten disorder realizations. It is indeed seen that the ratio is nearly constant around $\tau=\beta / 2$.

The frequency integral may now be evaluated analytically, leading to the following result:

$$
\sigma_{\mathrm{dc}}=\frac{\beta^{2}}{\pi} \Lambda_{x x}(\mathbf{q}=0, \tau=\beta / 2) .
$$

This approximation is expected to be valid when the temperature is smaller than an appropriate energy scale in the problem. It is convenient because it allows the computation of $\sigma_{\mathrm{dc}}$ as a function of temperature to be obtained from the function $\Lambda_{x x}(\mathbf{q}, \tau)$, which is calculated directly in DQMC.

Obtaining a transport property such as $\sigma_{\mathrm{dc}}$ directly from imaginary-time data, as described above, is a process which must be undertaken with caution. However, the use of this procedure gives the correct characterization as a metal or insulator in all cases, which we have checked so far. For example, $d \sigma_{\mathrm{dc}} / d T$ is positive (insulating behavior) for the half-filled $d=2$ Hubbard model without randomness at all values of $U$, that is, regardless of whether the insulating character arises predominantly from antiferromagnetic order (weak coupling) or Mott behavior (strong coupling). ${ }^{11,18}$ The procedure also gives the correct physics in a band insulator when a staggered site energy is present and $U=0$. It has also been shown to give the correct physics of the disordered attractive Hubbard model. ${ }^{16}$

A fundamental check of the numerical data is the verification that the longitudinal current-current correlation function obeys the gauge invariance condition

$$
\Lambda_{x x}\left(q_{x} \rightarrow 0, q_{y}=0, i \omega_{n}=0\right)=\mathcal{K},
$$

where $\mathcal{K}$ is the kinetic energy. We have checked that as in previous work, ${ }^{11,16,17}$ this condition is satisfied.

Since the disordered site energies in the system are generated randomly from a uniform distribution $\left(-\frac{1}{2} \Delta,+\frac{1}{2} \Delta\right)$, the distribution has zero mean and a variance

$$
\mathcal{V}_{0}^{2}=\frac{1}{\Delta} \int_{-\Delta / 2}^{\Delta / 2} \epsilon^{2} d \epsilon=\frac{\Delta^{2}}{12} .
$$

In order to study the screening of the disordered potential by interactions, we note that within a mean-field picture, an electron moving in the one-body potential $\epsilon_{j}$ will feel the site energy renormalized by the density of oppositely oriented electrons. That is,

$$
\tilde{\epsilon}_{j, \sigma}=\epsilon_{j}+U\left\langle n_{j,-\sigma}\right\rangle,
$$

which becomes, in the absence of spin polarization,

$$
\tilde{\epsilon}_{j}=\epsilon_{j}+\frac{U}{2}\left\langle n_{j}\right\rangle,
$$

since for each spin species $\left\langle n_{j \sigma}\right\rangle=\frac{1}{2}\left\langle n_{j}\right\rangle$. We define an associated dimensionless variance by normalizing to the fluctuations in the original site energies,

$$
\mathcal{V}^{2}=\frac{1}{\mathcal{V}_{0}^{2}}\left(\left\langle\widetilde{\epsilon}_{j}^{2}\right\rangle-\left\langle\widetilde{\epsilon}_{j}\right\rangle^{2}\right)=\frac{12}{\Delta^{2}}\left(\left\langle\widetilde{\epsilon}_{j}^{2}\right\rangle-\left\langle\widetilde{\epsilon}_{j}\right\rangle^{2}\right)
$$

In the absence of interactions $(U=0)$ or for very large $\Delta$ at fixed $U$, we have $\mathcal{V}=1$, indicating that there is no screening of the random potential. The question we wish to address is whether there is some signal, e.g., a noticeable decrease, in $\mathcal{V}$ upon entry into a metallic phase.

\section{METALLIC PHASE DUE TO INTERACTIONS}

We begin by demonstrating that interactions can drive an Anderson insulating phase metallic. We show in Fig. 2 the dc 


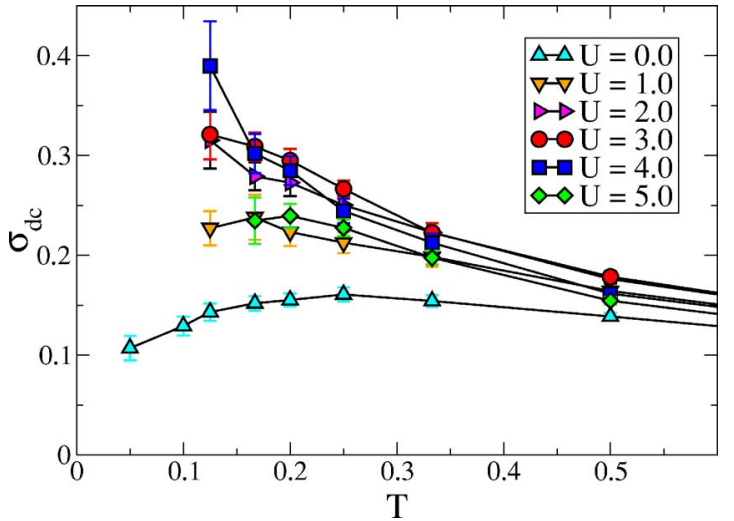

FIG. 2. (Color online) The dc conductivity as a function of temperature for increasing values of the on-site repulsion $U=0-5$. The site-energy variance $\Delta=8$.

conductivity as a function of temperature for a fixed strength of the disorder potential and increasing $U$. At $U=0, \sigma_{\mathrm{dc}}$ decreases as $T$ is lowered, indicating insulating behavior. However, at strong coupling, $\sigma_{\mathrm{dc}}$ increases as $T$ is lowered, indicating a crossover to metallic behavior. All results in Fig. 2 and subsequently in this paper, are at one-quarter filling $\rho=\langle n\rangle=\frac{1}{2}$. This is far from the most dramatic effects of $U$ in the Hubbard model - the Mott transition and antiferromagnetic ordering. The DQMC computations are carried out on a square lattice with linear size 8 .

The metallic phase in Fig. 2 can caused to return to insulating behavior by increasing the site disorder. This is shown in Fig. 3, where we begin with the interaction strength which gives the largest conductivity, $U=4$, and makes $\Delta$ larger. For $9<\Delta<10$, the low-temperature slope of $\sigma_{\mathrm{dc}}$ reverts to insulating character.

An interesting feature of Fig. 2 is the nonmonotonic behavior of the conductivity. $\sigma_{\mathrm{dc}}$ increases with $U$ up to $U \approx 3-4$ but then decreases again at $U=5$. In order to verify that this phenomenon is generic, we show in Fig. 4 data for larger $\Delta=9$. We again see that $\sigma_{\mathrm{dc}}$ comes down at strong coupling. A similar phenomenon occurs in the evolution of the superfluid density $\rho_{s}$ for correlated bosons moving in a

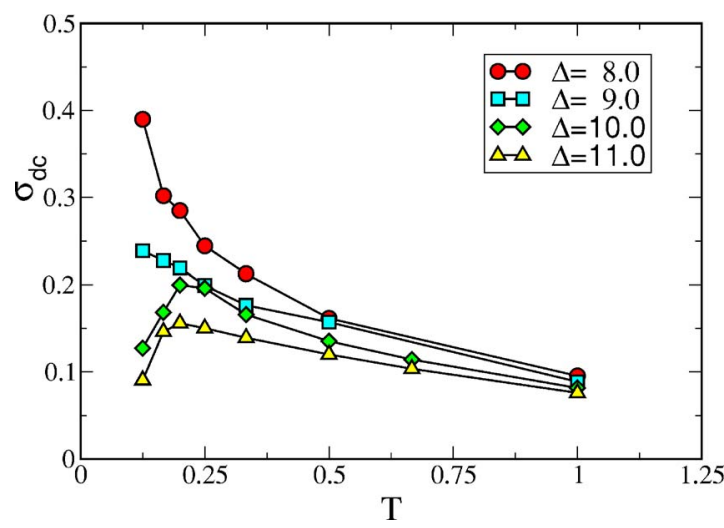

FIG. 3. (Color online) The de conductivity as a function of temperature for increasing values of disorder $\Delta=8-11$. The on-site repulsion $U=4$.

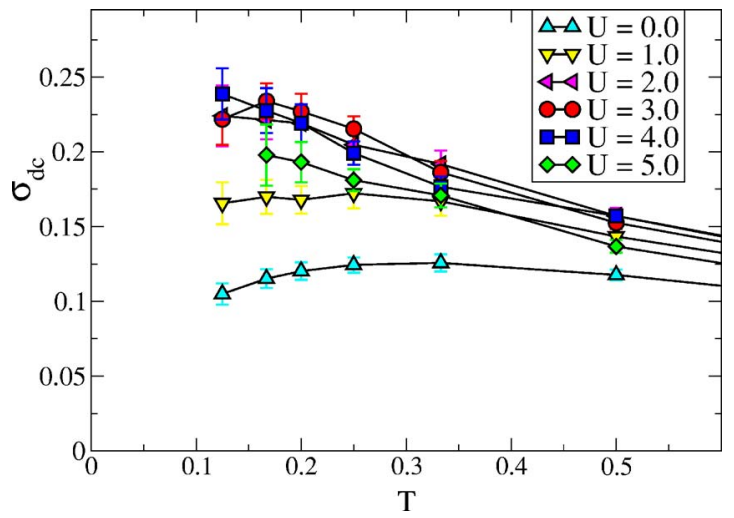

FIG. 4. (Color online) As in Fig. 2, except at larger disorder, $\Delta=9$. The same decrease of conductivity with $U$ in the metallic phase is seen as in Fig. 2.

random potential $-\mathrm{a}$ superfluid phase with $\rho_{s} \neq 0$ exists at intermediate coupling, but the system is insulating, $\rho_{s}=0$, both at weak and strong couplings. ${ }^{5}$

In Fig. 5, we show $\sigma_{\mathrm{dc}}$ vs the disorder strength for progressively lower temperature values. For $\Delta<\Delta_{c}$, the system is metallic and $\sigma_{\mathrm{dc}}$ increases as the temperature is lowered, while for $\Delta>\Delta_{c}$, in the insulating state, the behavior is opposite. The crossing point of the plots demarcates the critical disorder strength.

In Fig. 6, we show a similar crossing plot for $\sigma_{\mathrm{dc}}$ as we tune the interaction strength through the metal-insulator transition for a fixed disorder strength $\Delta=8$ (see Fig. 2). A small value of the interaction is seen to be enough to cause the transition to a metal. Interestingly, the conductivity is nonmonotonic and decreases for large values of the interaction strength (the fermion sign problem in DQMC simulations forbids the evaluation of $\sigma_{\mathrm{dc}}$ at $U=5, \beta=8$ ). It is possible that there is a crossing at larger interaction strengths when the system reverts back to an insulator. Such nonmonotonic behavior of the conductivity has also been seen in recent DQMC studies of a multiband Hubbard model at half-filling, where the sequence of transitions with increasing $U$ is found to be band insulator $\rightarrow$ metal $\rightarrow$ Mott insulator.

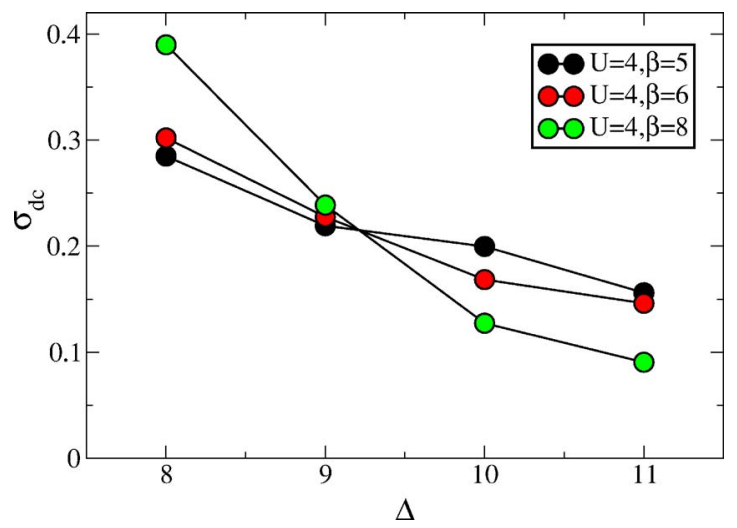

FIG. 5. (Color online) A crossing plot for $\sigma_{\mathrm{dc}}$ vs $\Delta$. The critical disorder strength $\Delta_{c} \sim 9.2-9.3$ for $U=4$ is clearly seen. 


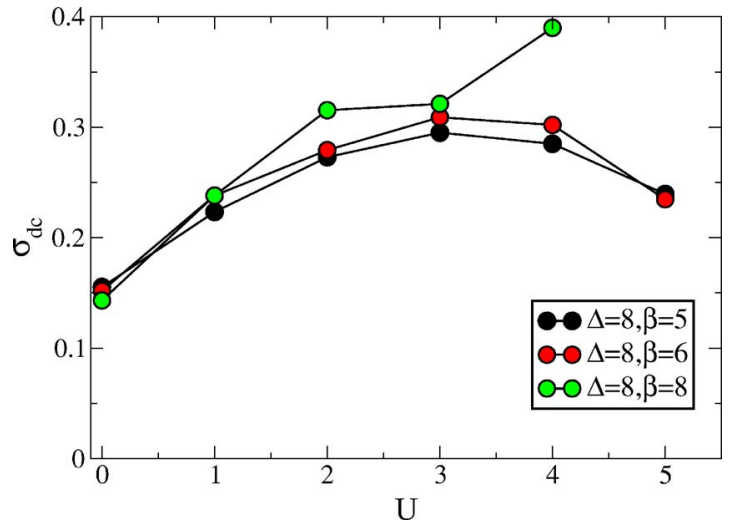

FIG. 6. (Color online) A crossing plot for $\sigma_{\mathrm{dc}}$ vs $U$. The crossing is seen to happen for $0<U<1$ for $\Delta=8$.

\section{RENORMALIZED SITE ENERGIES WITH INTERACTIONS}

Before showing the results for the dimensionless variance of the renormalized site energies, we present in Fig. 7 a plot of the original and renormalized site-energy landscapes. As expected, the particles preferentially sit on the sites with low $\epsilon_{j}$, and these larger values of $\left\langle n_{j}\right\rangle$ then lead to a smoother $\tilde{\epsilon}_{j}=\epsilon_{j}+\frac{U}{2}\left\langle n_{j}\right\rangle$. However, there is certainly no very dramatic leveling of the landscape. Below, we will explore this more quantitatively.

In Fig. 8, we examine whether there is signal of the metalinsulator transition in the evolution of $\mathcal{V}$. We plot the low- temperature slope $d \sigma_{\mathrm{dc}} / d T$ from the data of Fig. 3 and show its change of sign at $\Delta \approx 9.2-9.3$. There is no clear indication of this critical value in the renormalized site-energy variance $\mathcal{V}$.

We can similarly look for this effect at the metal-insulator transition driven by increasing $U$ at fixed $\Delta=8$ (Fig. 2). This is shown in Fig. 9. Again, there appears to be no clear signal of the metal-insulator transition in the screened site energies.

\section{RENORMALIZED SITE-ENERGIES WITH ZERO HOPPING}

The results from the previous section suggest that we look more closely at the physical picture of the smoothening of the site-energy landscape by interactions. Our expectation in Fig. 8, where we plotted $\mathcal{V}$ as a function of the disorder strength, was that in the metallic phase at weak disorder, there would be a markedly smaller value of $\mathcal{V}$ and then a crossover to a larger value as the disorder is increased into an insulating phase. On the other hand, at weak disorder, we expect the least inhomogeneity in the site occupations. In the limit of uniform density, at very weak disorder, the siteenergy variance equals the original one, and $\mathcal{V}^{2}=1$, suggesting that $\mathcal{V}$ might instead decrease with disorder. The conflicting tendency to decrease with disorder as charge inhomogeneity develops and increase in the insulating phase might explain why the site-energy variance is so insensitive to site-energy disorder, whereas when we tune through the transition with interaction strength, there is a much larger
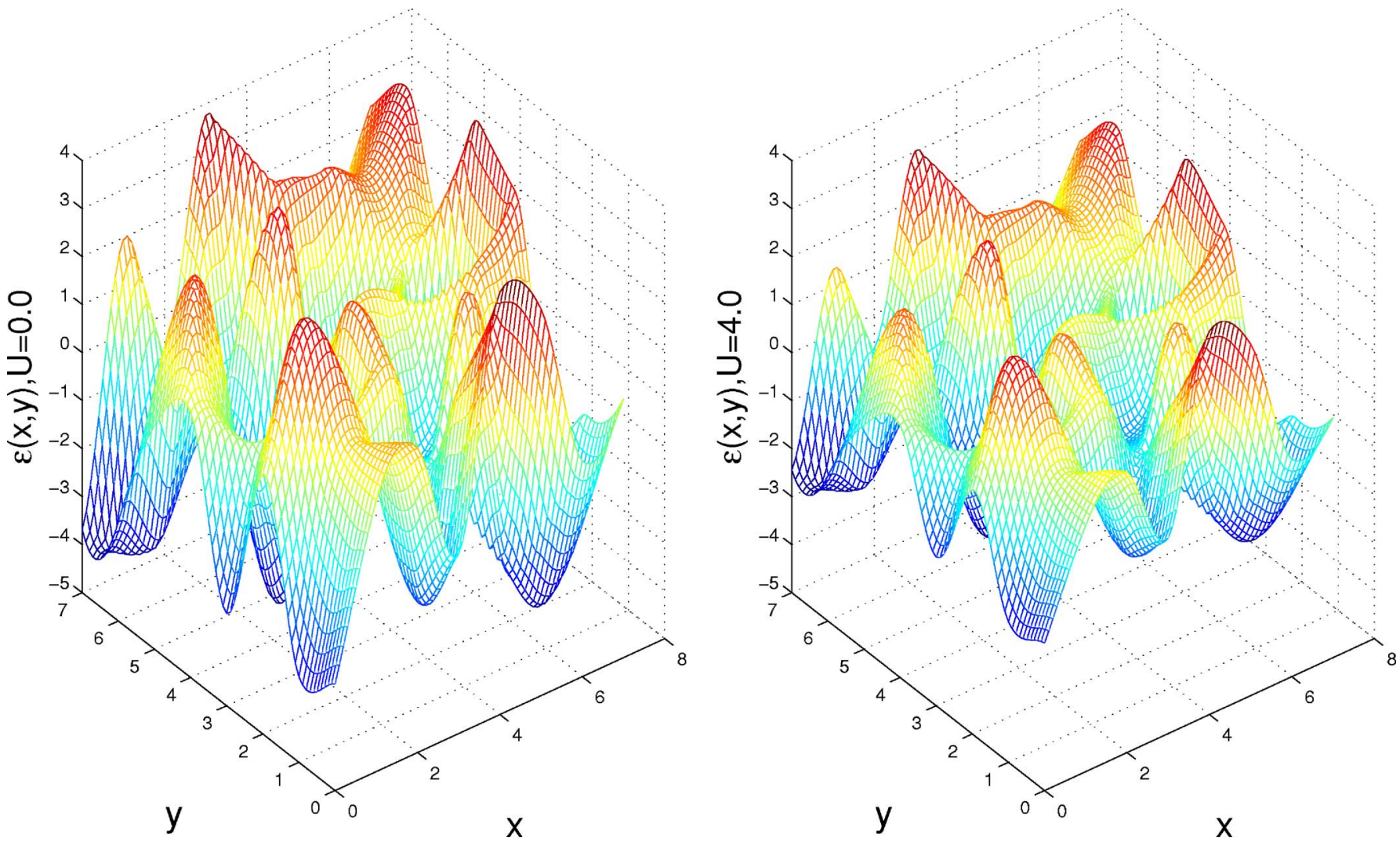

FIG. 7. (Color online) Left: Landscape of the original site energies $\epsilon_{j}$ with $\Delta=8$. Right: Landscape of the renormalized site energies $\tilde{\epsilon}_{j}$ with $\Delta=8, U=4$, and $\beta=8$. On the right, the mean increase in the renormalized site energies due to $U$ has been subtracted out. 


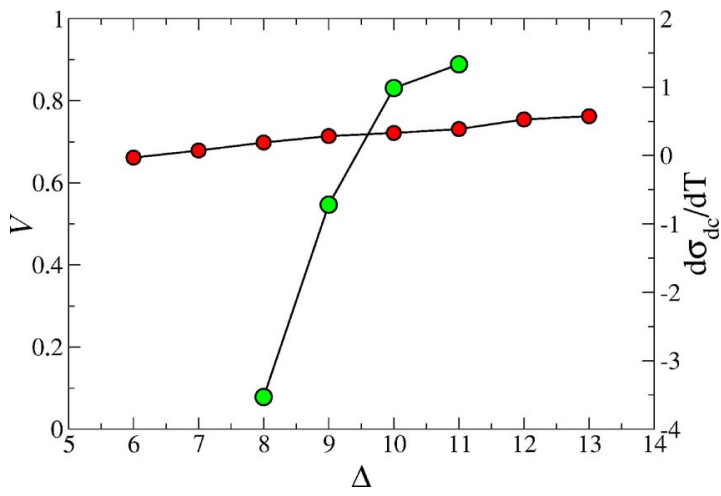

FIG. 8. (Color online) The variance of the renormalized site energy $\mathcal{V}$ is shown as a function of $\Delta$, as is the low-temperature slope of the conductivity $(U=4, \beta=8)$.

decline (though still no abrupt signal at the transition). In this section, we examine an analytically solvable limit of the disordered Anderson-Hubbard model, that of $t=0$, which can be considered to be the limit of very high disorder. At this limit, there is no metallic behavior, but it is still interesting to investigate the behavior of the site-energy distribution as we move from weak to strong interaction (or strong to weak disorder).

When there is no hopping, the Anderson-Hubbard model is classical. As electrons are added to the lattice at $t=0$, the sites with the lowest site energies are singly occupied up to the Fermi energy $E_{F}$. When, however, $E_{F}$ exceeds $-\Delta / 2+U$, it becomes preferable to start doubly occupying the lowenergy sites. This is illustrated in Fig. 10. From the figure, it is evident that $\left\langle n_{\uparrow}\right\rangle=\frac{1}{\Delta}\left(E_{F}+\Delta / 2\right)$ and $\left\langle n_{\downarrow}\right\rangle=\frac{1}{\Delta}\left(E_{F}+\Delta / 2-U\right)$ and hence that $\langle n\rangle=\left\langle n_{\uparrow}+n_{\downarrow}\right\rangle=\frac{1}{\Delta}\left(2 E_{F}+\Delta-U\right)$. We can easily obtain the mean of the renormalized site energies by averaging Eq. (9): $\left\langle\widetilde{\boldsymbol{\epsilon}}_{\uparrow}\right\rangle+\left\langle\widetilde{\boldsymbol{\epsilon}}_{\downarrow}\right\rangle=U\left(\left\langle n_{\uparrow}\right\rangle+\left\langle n_{\downarrow}\right\rangle\right)=\frac{U}{\Delta}\left(2 E_{F}+\Delta-U\right)$.

A completely equivalent result is obtained by recognizing that the energies of the sites on which up spin electrons reside are raised by $U$ in the range from $-\Delta / 2$ to $E_{F}-U$, where down spins are present. Similarly, the energies of the sites on which down-spin electrons reside are raised by $U$ in the range from $-\Delta / 2$ to $E_{F}$, where up spins are present. (Here,

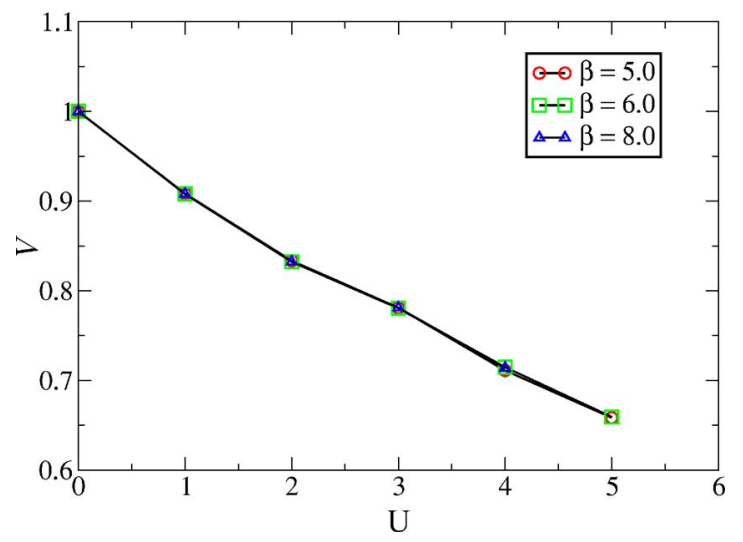

FIG. 9. (Color online) The renormalized site energy is shown as a function of $U$. There appears to be no signal of the MIT at small $U$ nor the conductivity peak at $U \approx 3-4$.

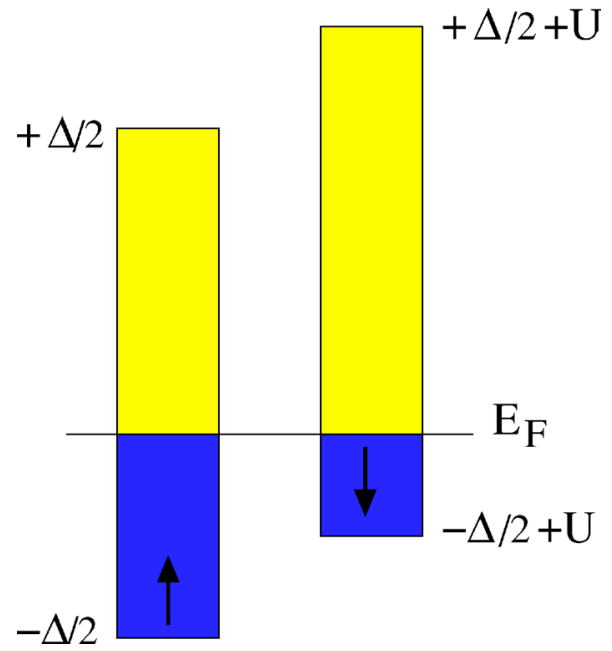

FIG. 10. (Color online) At $t=0$, the energy levels for occupation by the first electrons (which we denote by $\uparrow$ ) extend from $-\Delta / 2$ to $\Delta / 2$ (left). The energy levels for occupation by the second electrons (which we denote by $\downarrow$ ) extend from $-\Delta / 2+U$ to $+\Delta / 2+U$ (right).

the designations "up" and "down" merely reflect the "first" and "second" electrons on a site.) When the energies are averaged over these ranges, the same result for $\left\langle\tilde{\boldsymbol{\epsilon}}_{\uparrow}\right\rangle+\left\langle\tilde{\boldsymbol{\epsilon}}_{\downarrow}\right\rangle$ is obtained.

The average of the square of the renormalized site energies is obtained in the same way. We can then evaluate the dimensionless variance of the renormalized site energies $\mathcal{V}_{t=0}^{2}$, defined as

$$
\mathcal{V}_{t=0}^{2}=\frac{12}{\Delta^{2}}\left(\left\langle\tilde{\epsilon}_{j}^{2}\right\rangle-\left\langle\tilde{\epsilon}_{j}\right\rangle^{2}\right)
$$

To determine the variance, we must distinguish between two cases: a generic one, in which the Fermi energy $E_{F}$ is larger than $-\Delta / 2+U$ and there is double occupancy of the lowenergy sites, and a nongeneric case, in which there is only single occupancy, which may happen for a large value of $x$ $=U / \Delta$ or a small density. For the generic case, the variance can be computed in terms of the three energy scales $(U, \Delta$, and $\left.E_{F}\right)$ in the $t=0$ problem:

$$
\begin{aligned}
\mathcal{V}_{t=0}^{2}= & 1+\frac{3 U^{2}}{\Delta^{2}}-\frac{3 U}{\Delta}-\frac{3 U^{4}}{\Delta^{4}}+\frac{6 U^{3}}{\Delta^{3}}+\frac{12 E_{F}^{2} U}{\Delta^{3}}+\frac{12 E_{F} U^{3}}{\Delta^{4}} \\
& -\frac{12 E_{F} U^{2}}{\Delta^{3}}-\frac{12 E_{F}^{2} U^{2}}{\Delta^{4}} .
\end{aligned}
$$

The Fermi energy (for the generic case) can be determined in terms of $U$ and $\Delta$ by the following equation:

$$
\rho=\frac{E_{F}+\frac{\Delta}{2}}{\Delta}+\frac{E_{F}+\frac{\Delta}{2}-U}{\Delta},
$$

where $\rho$ is the filling. For example, in the quarter-filled case, $\rho=\frac{1}{2}$ and $E_{F}=\frac{1}{2}\left(U-\frac{\Delta}{2}\right)$. In the nongeneric case, the Fermi energy can, of course, be determined by simple state counting. 


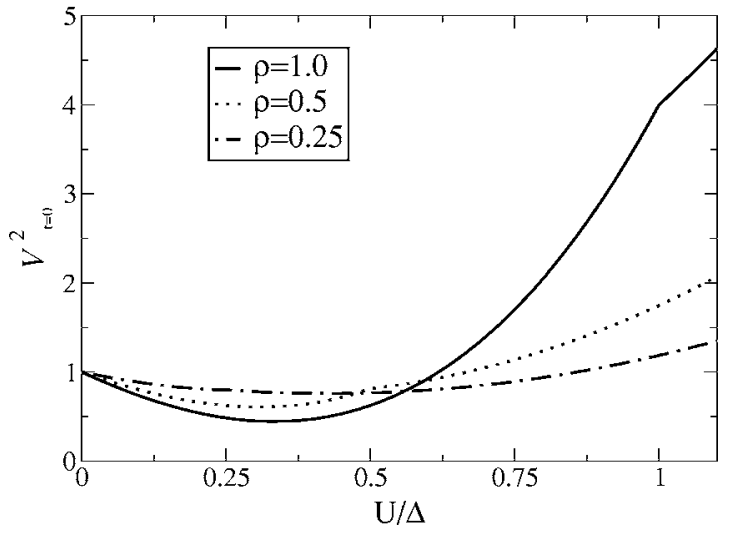

FIG. 11. The site-energy variance, as defined in Eq. (12), is plotted for three different values of the filling, $\rho=1,0.5$, and 0.25 . The $x$ axis is the ratio of the interaction to the disorder strength, $U / \Delta$.

A plot of $\mathcal{V}_{t=0}^{2}$ vs $x=U / \Delta$ is given in Fig. 11 for different fillings. At very weak interaction (or very strong disorder), the variance equals the noninteracting value 1 . As the interaction is increased (equivalently, the disorder is decreased), the variance first decreases and reaches a minimum. Upon further increasing the interaction, however, the site occupations become homogeneous and the variance grows.

\section{CONCLUSIONS}

We have examined the metal-insulator transition in the Anderson-Hubbard model using determinant quantum Monte Carlo simulations. Our focus has been on the evolution of the renormalized site energy through the transition, and we conclude that it exhibits no sharp feature there. It seems that the picture of screening of the disorder by interaction is too primitive to account for metallic behavior.

On the other hand, we observe an interesting nonmonotonic behavior of the conductivity with interaction strength. In the boson Anderson-Hubbard model, the ground state at incommensurate densities is an Anderson insulator at weak $U$ and an insulating "Bose glass" at large $U$. In between, there is a superfluid phase in which the superfluid density first rises, as one emerges from the Anderson insulator, and then falls to zero again upon entry into the Bose glass. As far as we can see, the fermion Hubbard model remains metallic at large $U$, but simulations there are difficult and we cannot make a definitive statement. In any case, the nonmonotonic behavior of $\sigma_{\mathrm{dc}}$ is rather analogous to the behavior seen for strongly interacting, disordered Bose systems.

In this paper, we have looked at site-energy renormalization defined in the framework of the Hartree-Fock approximation away from half-filling [see Eq. (9)]. A previous study ${ }^{19}$ found much stronger screening of the renormalized site energies in the Mott metal-insulator transition at halffilling using a more general definition of the renormalized site energies involving the full self-energy of the system. The self-energy could be obtained using the approximate dynamical mean-field theory. It will be a subject of future research to explore the effect on screening of the site energies by taking into account correlations via the full self-energy as computed using the determinant QMC method.

\section{ACKNOWLEDGMENTS}

We acknowledge support from the National Science Foundation under Awards No. NSF DMR 0312261 and NSF DMR 0421810. We are grateful to W. E. Pickett, B. Altman, and V. Dobrosavljevic for useful discussions.
${ }^{1}$ P. A. Lee and T. V. Ramakrishnan, Rev. Mod. Phys. 57, 287 (1985); D. Belitz and T. R. Kirkpatrick, ibid. 66, 261 (1994).

${ }^{2}$ S. V. Kravchenko and M. P. Sarachik, Rep. Prog. Phys. 67, 1 (2004); E. Abrahams, S. V. Kravchenko, and M. P. Sarachik, Rev. Mod. Phys. 73, 251 (2001), and references therein.

${ }^{3}$ A. Punnoose and A. M. Finkelstein, Science 310, 289 (2005); S. Anissimova, S. V. Kravchenko, A. Punnoose, A. M. Finkelstein, and T. M. Klapwijk, cond-mat/0609181 (unpublished).

${ }^{4}$ M. P. A. Fisher, P. B. Weichman, G. Grinstein, and D. S. Fisher, Phys. Rev. B 40, 546 (1989).

${ }^{5}$ R. T. Scalettar, G. G. Batrouni, and G. T. Zimanyi, Phys. Rev. Lett. 66, 3144 (1991).

${ }^{6}$ W. Krauth, N. Trivedi, and D. M. Ceperley, Phys. Rev. Lett. 67, 2307 (1991).

${ }^{7}$ M. C. O. Aguiar, V. Dobrosavljevic, E. Abrahams, and G. Kotliar, Phys. Rev. B 71, 205115 (2005).

${ }^{8}$ K. Byczuk, M. Ulmke, and D. Vollhardt, Phys. Rev. Lett. 90, 196403 (2003).

${ }^{9}$ K. Byczuk, W. Hofstetter, and D. Vollhardt, Phys. Rev. B 69, 045112 (2004).
${ }^{10}$ A. Garg, H. R. Krishnamurthy, and M. Randeria, Phys. Rev. Lett. 97, 046403 (2006).

${ }^{11}$ P. J. H. Denteneer, R. T. Scalettar, and N. Trivedi, Phys. Rev. Lett. 83, 4610 (1999).

${ }^{12}$ P. J. H. Denteneer and R. T. Scalettar, Phys. Rev. Lett. 90, 246401 (2003).

${ }^{13}$ Ji-Woo Lee, S. Chandrasekharan, and H. U. Baranger, Phys. Rev. B 72, 024525 (2005).

${ }^{14}$ R. Blankenbecler, D. J. Scalapino, and R. L. Sugar, Phys. Rev. D 24, 2278 (1981).

${ }^{15}$ J. E. Hirsch, Phys. Rev. B 31, 4403 (1985).

${ }^{16}$ N. Trivedi, R. T. Scalettar, and M. Randeria, Phys. Rev. B 54, R3756 (1996).

${ }^{17}$ D. J. Scalapino, S. R. White, and S. C. Zhang, Phys. Rev. B 47, 7995 (1993).

${ }^{18}$ N. Paris, K. Bouadim, F. Hebert, G. G. Batrouni, and R. T. Scalettar, Phys. Rev. Lett. 98, 046403 (2007).

${ }^{19}$ D. Tanaskovic, V. Dobrosavljevic, E. Abrahams, and G. Kotliar, Phys. Rev. Lett. 91, 066603 (2003). 